\title{
Optimization of F2 Layer Parameters Using IRI-Plas and IONOLAB-TEC
}

\author{
Oktay ŞAHIN ${ }^{1}, \underline{\text { Umut SEZEN }}^{2}$, Feza ARIKAN ${ }^{2}$, Orhan ARIKAN \\ ${ }^{1}$ Aselsan A.Ş. \\ oksahin@aselsan.com.tr \\ ${ }^{2}$ Hacettepe University, Department of Electrical and Electronics Engineering, Beytepe, Ankara 06800, Turkey \\ u.sezen@ee.hacettepe.edu.tr, $\underline{\operatorname{arikan} @ \text {,hacettepe.edu.tr, }}$ \\ ${ }^{3}$ Bilkent University, Department of Electrical and Electronics Engineering, Bilkent, Ankara 06800, Turkey \\ oarikan@ee.bilkent.edu.tr
}

\begin{abstract}
In this study, the relation of the maximum ionization height (HmF2) and the critical frequency (FoF2) of F2 layer is examined within their parametric range through the International Reference Ionosphere extended towards the plasmasphere (IRI-Plas) model and the IONOLAB-TEC. HmF2 and FoF2 are optimized using an iterational loop through Non-Linear Least Squares method. HmF2 and FoF2 are obtained for various locations including Turkey for the same quiet day. Results are compared with ionosonde data where available. This study enables the modification and update of empirical and deterministic IRI Model to include instantaneous variability of the ionosphere.
\end{abstract}

\section{Introduction}

Ionosphere is the layer of the atmosphere that lies between $60 \mathrm{~km}$ and $1000 \mathrm{~km}$ above Earth surface. Ionosphere has a great importance in high frequency (HF) and satellite communications because of its electrical and ionic structure. Its ionization characteristics and electron density change according to the location on Earth, time, Sun, geomagnetic and seismic effects. Ionosphere consists of three distinct layers, namely, D, E and F. F layer can be divided into F1 and F2 layers. F2 layer, having the highest electron density, is the most stable layer for HF communication and it has major importance in satellite communications [1]. Many experimental and theoretical models have been investigated for obtaining a realistic physical structure of ionosphere. The most significant and commonly used one of these models is International Reference Ionosphere (IRI) Model [2]. IRI is an international project sponsored by the Committee on Space Research (COSPAR) and the International Union of Radio Science (URSI). For a given location, time and date, IRI provides monthly averages of the electron density, electron temperature, ion temperature, and ion composition (www.iri.org). In a recent study, IRI model is extended to include plasmasphere (IRI-Plas) upto the height of $20,000 \mathrm{~km}$ corresponding to the height of GPS satellites [3]. In IRI-Plas, TEC estimates can be provided externally for scaling of topside and plasmasphere extensions. In this study, GPS-TEC is obtained as IONOLAB-TEC [4-5] and provided externally for scaling of F2 layer parameters. The goal of this study is to update IRI-Plas with IONOLAB-TEC in an iterative optimization loop formed by Non-Linear Least Squares method. In this study, IONOLAB TEC method is shortly defined in Section 2. IRI-Plas model is defined in Section 3 and optimization method for finding the F2 layer parameters is defined in Section 4.

\section{TEC Estimations Using lonolab Method}

IGS analysis centers can produce TEC estimations, but resolution of these estimates is low or they depend on observational data availability. In this study, GPS-TEC data is obtained from www.ionolab.org as IONOLAB-TEC [4-6]. IONOLAB-TEC combines data from all the GPS satellites that are above $10^{\circ}$ elevation angle (horizon limit) of the GPS station with a temporal resolution of 30 seconds. The method calculates VTEC (Vertical Total Electron Content) per satellite and combines them using a weighting function based on satellite positions which reduces the contamination caused by multipath effects [5-6]. The receiver differential code bias are estimated using the method described in [4].

\section{IRI-Plas Model}

IRI-Plas Model is an ionospheric empirical model that can to be updated with different observational data sources [3]. In IRI-Plas, the region of interest can be extended to plasmasphere, i.e. $20,000 \mathrm{~km}$. Ion and electron density distributions and TEC estimates along the local zenith axis can be obtained using this model. The model 
computations can be scaled by the input of external parameters such as GPS-TEC. Some of the model input parameters, such as sun spot number and geomagnetic coordinates are non-adaptive and kept constant in the data set. Some parameters are option selection flags such as FoF2 and TEC. Output parameters of the model are layer TEC estimates and related critical values. In this study, HmF2, FoF2 and TEC are investigated as input parameters. If we define parameter $h$ as $\mathrm{HmF} 2, f$ as critical frequency of the signal in layer, $\mathbf{y}$ as IONOLABTEC data of that hour and $\mathbf{x}$ vector as non-optimized parameters such as selected receiver latitude, longitude, date, time, daily sun spot number and $\mathrm{K}_{\mathrm{p}}$ index (Sun spot number and $\mathrm{K}_{\mathrm{p}}$ index are database inputs independent of user entries), model finds estimation vector $\hat{\mathbf{y}}$ as given:

$$
\hat{\mathbf{y}}=\operatorname{iriplas}(h, f, \mathbf{x}, \mathbf{y})
$$

\section{Determination of F2 Layer Parameters}

During day light, all ionosphere layers appear but when ionization effect of Sun disappears at nights, only F2 layer remains in spite of its decreasing electron density profile. So F2 layer is needed to be carefully examined. Main two characterizing parameters of this layer is $\mathrm{HmF} 2(\mathrm{~km})$ and FoF2 (MHz). Only electrical signals above a critical frequency level can traverse the ionosphere and propagate into outer space. Signals with lower frequencies are refracted and reflected. Therefore, FoF2 is very important in radio communication. IRIPlas model, estimates critical frequency and height values by analyzing its preset coefficient matrices. If IRIPlas is updated with GPS-TEC, more realistic HmF2 and FoF2 estimates can be obtained. The iterational optimization loop model for minimizing the TEC error is provided in Figure 1.

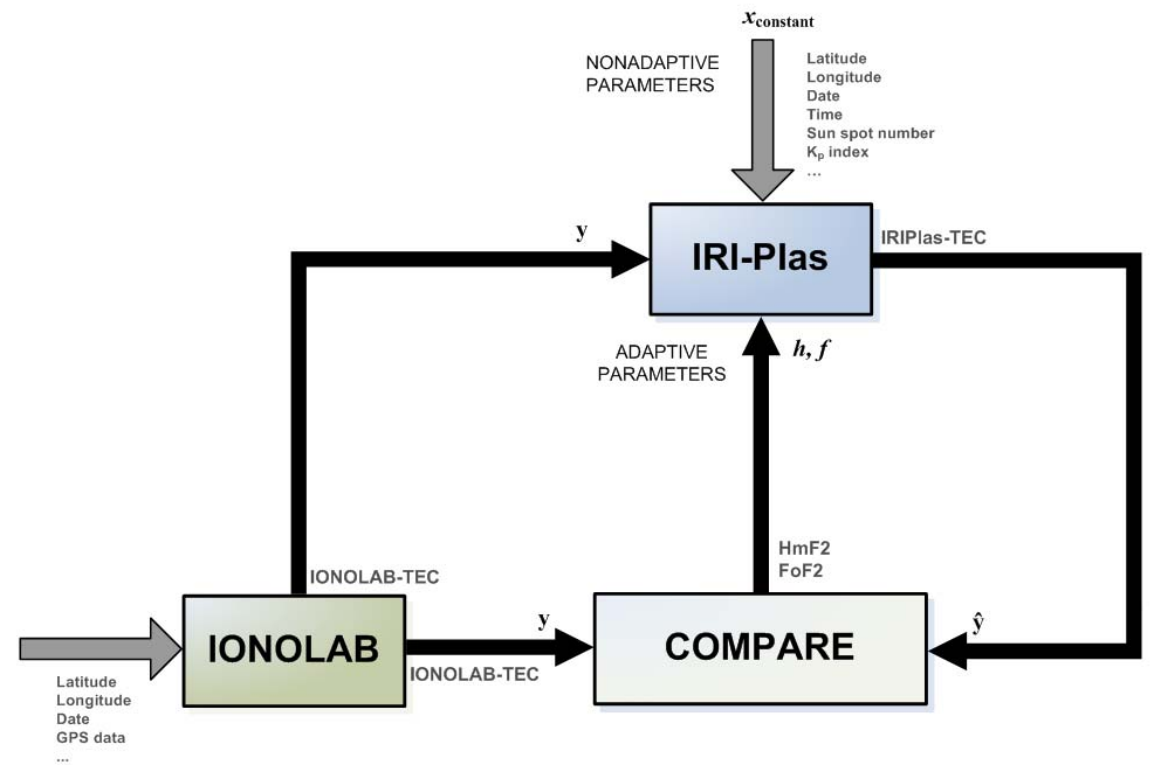

Figure 1: IRI-Plas Iterative Optimization Model

Defining error vector $\mathbf{e}$ as the difference of observational data set $\mathbf{y}$ and IRI-Plas TEC estimations $\hat{\mathbf{y}}$ for each hour of day,

$$
\mathbf{e}=\mathbf{y}-\hat{\mathbf{y}}
$$

and minimizing error norm $\|\boldsymbol{e}\|$ in an iterative loop leads us to optimized $h$ and $f$ parameters.

Norm characteristics of daily TEC error vector is investigated in 150-500 km parametric range of HmF2 with $50 \mathrm{~km}$ stepsize and 4-9 MHz parametric range of FoF2 with $0.5 \mathrm{MHz}$ step size separately and results are shown on Figure 2. For HmF2 cost function analysis, FoF2 is taken as a constant average value which is $6 \mathrm{MHz}$. By the same way for FoF2 cost function analysis, HmF2 is taken as a constant average value which is $250 \mathrm{~km}$. After examining of TEC error characteristics, it is seen that there are no local minima (only a global minimum). So, selecting Non-Linear Least Squares method for optimizing HmF2 and FoF2 parameters, initializing the algorithm with $\mathrm{HmF} 2$ and FoF2 estimations obtained from IRI-Plas model (without using IONOLAB TEC) and running algorithm with IONOLAB TEC updates would lead us to a meaningful solution.

It is clearly seen in Figure 3 that optimization leads us to a one-to-one matching TEC estimations with IONOLAB TEC observations. 

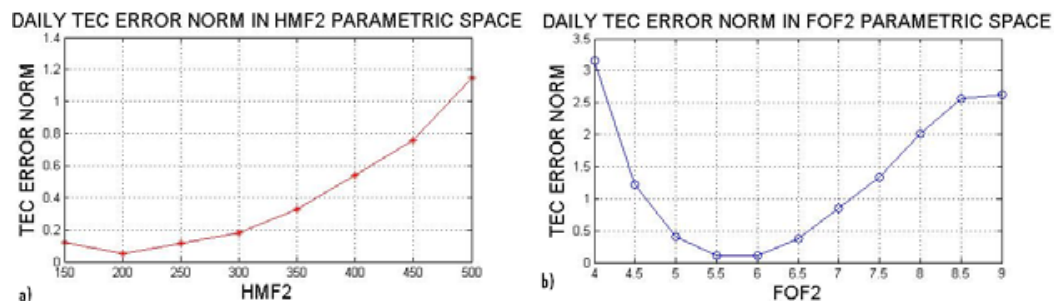

Figure 2: Ankara, 15 Oct 2008, quiet day daily TEC error norm plot for a) $\mathrm{HmF} 2$, b) FoF2 parametric range

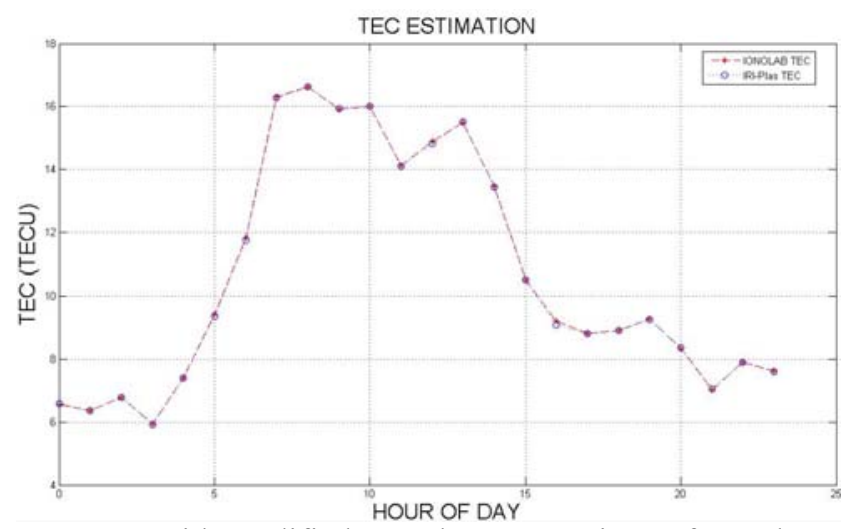

Figure 3: Comparison of IONOLAB-TEC with modified IRI-Plas TEC estimate for Ankara, 15 Oct 2008.

For selected day and IGS receiver, daily resulting TEC estimation error norm (using $\|\boldsymbol{e}\|$ ) for optimizing parameters is found as 4.5875e-007 TECU. Nearest ionosonde to Ankara is AT138 that is located in Athens, Greece on same latitudal coordinates. Comparison of $\mathrm{HmF} 2$ and FoF2 estimated parameters and ionosonde data for 15 Oct 2008, ankr (Ankara, Turkey) IGS receiver are given on Figure 4 below. Red lines are observational ionosonde data and blue ones are estimations.

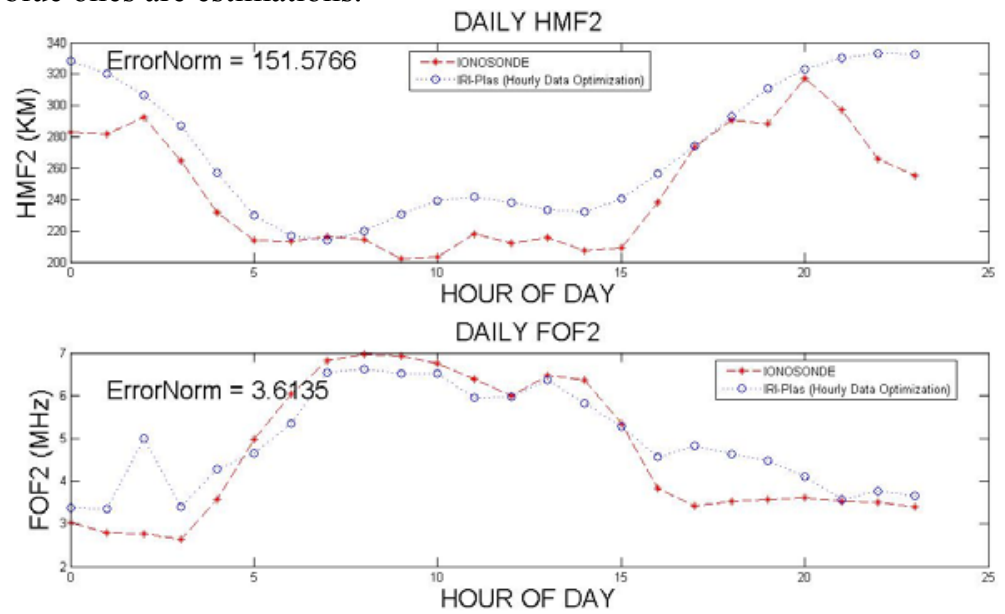

Figure 4: Comparison of estimation with ionosonde data for ankr - AT138 a) HmF2, b) FoF2

The HmF2 and FoF2 graphs matched well with the ionosonde data. Matching RMS errors of HmF2 and FoF2 are $151.58 \mathrm{~km}$ and $3.61 \mathrm{MHz}$, respectively. Although ankr and AT138 are not colocated, the error between the ionosonde data and modified IRI-Plas data are in very good agreement.

The optimization algorithm is tried for higher latitudes with closer ionosonde-IGS receiver locations. The results for wroc (Wroclaw) IGS receiver and nearest Juliusruh/Rugen (JR055) ionosonde are provided in Figure 5. Again, red lines denote the ionosonde data and the blue ones are estimates. Error norm is found as $117.62 \mathrm{~km}$ for $\mathrm{HmF} 2$ and $1.39 \mathrm{MHz}$ for $\mathrm{FoF} 2$. It is observed that with closer IGS station-ionosonde pair, the estimates are in better agreement with the experimental data. In Figure 6, the comparison is provided for karr (Karratha) IGS receiver and Learmonth (LM42B) ionosonde. On Figure 6, red lines are ionosonde data and the blue ones are the estimates. Error norm is found as $79.18 \mathrm{~km}$ for HmF2 and $2.15 \mathrm{MHz}$ for FoF2.An excellent agreement between the estimates and ionosonde data is obtained in this case also. 


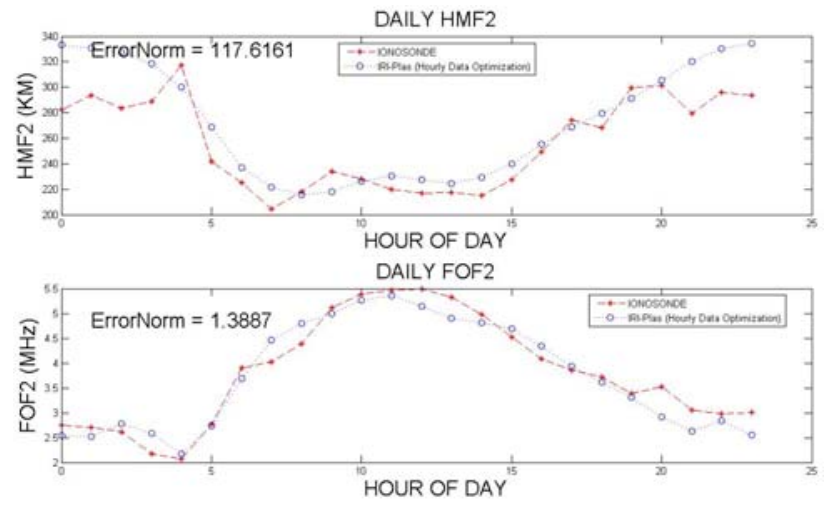

Figure 5: Comparison of estimation with ionosonde data for wroc - JR055 a) HmF2, b) FoF2

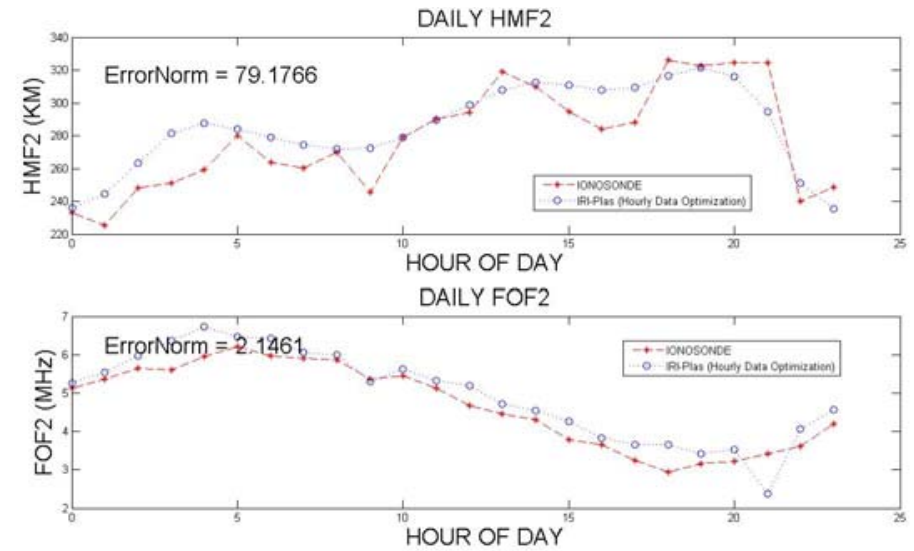

Figure 6: Comparison of estimation with ionosonde data for karr - LM42B a) HmF2, b) FoF2

\section{Conclusion}

IRI-Plas model is updated externally using IONOLAB-TEC estimates through an iterative optimization loop, and an updated HmF2 and FoF2 parameter set are obtained from IRI-Plas. These new set of FoF2 and $\mathrm{HmF} 2$ estimates from IRI-Plas are compared with nearest ionosonde data set and it is observed that the estimations were in excellent agreement with the ionosonde data. Estimation errors were higher for night hours.

From another perspective, these results also justify the reliability of the IRI-Plas model. This optimization method allow computation of the HmF2 and FoF2 parameters of the ionosphere F2 layer using the IRI-Plas model and GPS TEC estimations (or TEC measurements) where the ionosonde data are not available.

\section{Acknowledgments}

This study is supported by TUBITAK EEEAG Grant No: 109E055

\section{References}

1. M.O. Kolawole, Radar Systems, Peak Detection and Tracking, Oxford, UK: Newnes, 2002.

2. D. Bilitza (ed.), International Reference Ionosphere 1990, NSSDC 90-22, Greenbelt, Maryland, 1990.

3. T.L. Gulyaeva, "Storm time behavior of topside scale height inferred from the ionosphere-plasmasphere model driven by the F2 layer peak and GPS-TEC observations," Advances in Space Research, doi: 10.1016/j.asr.2010.10.025, 2010

4. F. Arikan, H. Nayir, U. Sezen and O. Arikan, "Estimation of single station interfrequency receiver bias using GPS-TEC", Radio Science , 43, RS4004, 2008, pp. 1-13.

5. H. Nayir, F. Arikan, O. Arikan, and C. B. Erol, "Total Electron Content Estimation with Reg-Est", J. Geophys. Res -Space Physics, 112(A11313), 2007, pp. 1-11.

6. F. Arikan, U. Sezen, O. Arikan,, O. Ugurlu, H. Nayir, "SpaceWeather Activities of IONOLAB Group: IONOLAB-TEC”, Geophysical Research Abstracts, 11:EGU2009-5188, 2009. 